MАТНЕМАТІCA, 62 (85), $\mathrm{N}^{\circ}$ 1, 2020, pp. 61-72

\title{
RANDOM GALOIS EXTENSIONS OF HILBERTIAN RINGS
}

\author{
MOSHE JARDEN and AHAROM RAZON
}

\begin{abstract}
Let $R$ be a countable Hilbertian ring with quotient field $K$ and let $L$ be a Galois extension of $K$. We generalize a result of Lior Bary-Soroker and Arno Fehm from fields to rings and prove that, for an abundance of large Galois extensions $N$ of $K$ within $L$, the integral closure of $R$ in $N$ is Hilbertian.
\end{abstract}

MSC 2010. 12E30

Key words. Hilbertian ring.

\section{REFERENCES}

[1] L. Bary-Soroker and A. Fehm, Random Galois extensions of Hilbertian rings, J. Théor. Nombres Bordeaux, 25 (2013), 31-42.

[2] M. Fried and M. Jarden, Field arithmetic, 3rd Edition, Ergebnisse der Mathematik, Vol. 11, Springer, Heidelberg, 2008.

[3] D. Haran, Hilbertian fields under separable algebraic extensions, Invent. Math., 137 (1999), 113-126.

[4] M. Jarden, Large normal extension of Hilbertian fields, Math. Z., 224 (1997), 555-565.

[5] M. Jarden and A. Razon, Extensions of Hilbertian rings, Glasg. Math. J., 62 (2018), $1-11$.

Received September 8, 2018

Tel Aviv University

Accepted November 4, 2018

School of Mathematics

Tel Aviv, Israel

E-mail: jarden@post.tau.ac.il

Elta Industry

Ashdod, Israel

E-mail: razona@elta.co.il

DOI: 10.24193/mathcluj.2020.1.07 